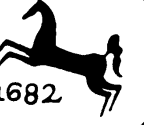

Sammlung Metzler Band 235 


$$
\text { Joachim Paech }
$$

\section{Literatur und Film}

J. B. Metzlersche Verlagsbuchhandlung

Stuttgart 


\section{Für Anne}

CIP-Titelaufnahme der Deutschen Bibliothek

Paech, Joachim:

Literatur und Film / Joachim Paech. -

Stuttgart: Metzler, 1988

(Sammlung Metzler; Bd. 235)

ISBN 978-3-476-10235-5

NE: GT

ISBN 978-3-476-10235-5

ISBN 978-3-476-03938-5 (eBook)

DOI 10.1007/978-3-476-03938-5

ISSN 0558-3667

\section{SM 235}

Dieses Werk einschließlich aller seiner Teile ist urheberrechtlich geschützt. Jede Verwertung außerhalb der engen Grenzen des Urheberrechtsgesetzes ist ohne Zustimmung des Verlages unzulässig und strafbar. Das gilt insbesondere für Vervielfältigungen, Übersetzungen, Mikroverfilmungen und die Einspeicherung und Verarbeitung in elektronischen Systemen.

(C) 1988 Springer-Verlag GmbH Deutschland

Ursprünglich erschienen bei J. B. Metzlersche Verlagsbuchhandlung und Carl

Ernst Poeschel Verlag GmbH in Stuttgart 1988 


\section{Inhalt}

Vorwort

1. Die Anfänge des Films in der populären Kultur .......

2. Die Institutionalisierung und Literarisierung des Films

3. Literaturgeschichte als Vorgeschichte des Films ....... 45

4. Kulturgeschichte als Vorgeschichte des Kinos

4.1 Die Eisenbahn

4.2 Das Panorama

4.3 Das Warenhaus

4.4 Fließband und Traumfabrik

5. Der literarische Film

6. Eine neue Literatur?

104

7. Filmische Schreibweise

8. Der ,,reine" und der , unreine" Film

8.1 Literarische und filmische Avantgarde ............... 152

8.2 Die Kunst des Sichtbaren? ..................................... 157

8.3 Der neue Realismus des Tonfilms ........................ 166

8.4 Literarische und filmische Schreibweise ............ 173

8.5 Der klassisch-realistische Text .............................. 176

9. Die literarische Lektüre eines Films ........................... 180

Literaturverzeichnis .......................................................... 207

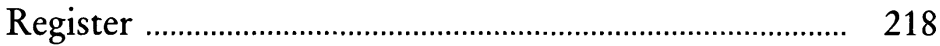




\section{Vorwort}

"Schließlich glaube ich, daß es das Größte ist, was es zu filmen gibt, Menschen sind, die lesen. « (Jean-Luc Godard)

Zur Erinnerung: Hans Robert Jauß hatte die Ursache für die von ihm konstatierte aktuelle Krise der Literaturwissenschaft in »einer neuen, noch nicht dagewesenen Herausforderung der Literatur als autonomer Kunst: die Herausforderung durch die Massenmedien [gesehen]. Methoden, die an der Höhenkammliteratur, am Kanon der klassischen, der nationalen oder wie immer bewerteten Gipfelwerke ausgebildet wurden, versagen vor der Wirkung der nicht-kanonischen Künste. Die Literatur [die diesem Kanon folgt und der in Hochschulen und Schulen weiterhin entsprochen wird], tritt in der Lebenspraxis völlig hinter dem zurück, was der Fernsehapparat vor Augen stellt, was sich der Normalverbraucher am Kiosk kauft, was uns alle in den ästhetisch oft raffinierten Formen der Werbung überflutet« (Jauß, 1969, S. 55).

Die Reaktion der Literaturwissenschaft auf die Herausforderung durch die Massenmedien ist durch einfaches Ignorieren und Katastrophenstimmung gekennzeichnet; Ignorieren bedeutet, daß noch immer Lehrer nicht systematisch für den technisch-methodischen Umgang mit audio-visuellen Medien aus-(fort-)gebildet werden, und daß Bemühungen, in das literaturwissenschaftliche Studium Filmgeschichte und -analyse, die kompetente Auseinandersetzung mit filmischen Adaptionen literarischer Werke, aufzunehmen, torpediert werden. Und Panik hat sich breitgemacht, wenn vom Ende der Schriftkultur und des Buches, vom (Fern-)Sehen statt (Buch-)Lesen die Rede ist und trotzig die »Urworte, orphisch « gegen die Unkultur der Bilder gesetzt werden.

Aber weder hat sich die "Furcht, die elektronischen Medien könnten eine buchlose Gesellschaft begründen «, bestätigt: "Die Akzeptanz und Nutzung des Mediums Buch wächst, wenn auch nur langsam, von Jahr zu Jahr « (Gruner + Jahr Branchenbild: Marktanalyse Nr. 21 Febr. 1983, S. 8). Noch wird sich die Vermeidungsstrategie gegenüber den Massenmedien durchhalten lassen: Der Paradigmawechsel zu einer Neubestimmung von Literatur, die unterschiedlichste (Kon-)Texte der medienvermittelten Kommunikation einbezieht, vollzieht sich längst; Produktion und Konsumtion von Literatur finden in allen Medien, die dafür in Frage kom- 
men, statt, im und mit dem Buch ebenso wie im und mit dem Kino, Fernsehen, Video etc. "Die Marquise von O.«, "Effi Briest«, "Die Buddenbrooks «, "Der Tod in Venedig «, "Der Zauberberg", "Mephisto « uva. sind literarische Buchtexte und Filme, die nebeneinander und einander ergänzend gelesen und gesehen werden.

Wenn Schulen und Hochschulen vor dieser Entwicklung die Augen verschließen und die Konsequenzen zu ziehen sich scheuen, dann auch deshalb, weil der literaturwissenschaftliche und -didaktische Umgang zum Beispiel mit Goethes »Wahlverwandtschaften « zwar sgekonnt< wird, Claude Chabrols gleichnamiger Film nach Goethes Roman jedoch organisatorisch und methodisch kaum bewältigt werden kann. Inzwischen gibt es gegenüber einer Vielzahl hervorragender französischer - z. B. die Studie von Francis Vanoye (1979) - und englisch/amerikanischer Beispiele auch in Deutschland einige Arbeiten zur Methodologie der Analyse von Filmen, speziell verfilmter Literatur (Vgl. die Einführungen in die Filmanalyse Faulstich, 1976; Kuchenbuch, 1978; Hickethier/Paech, 1979; Silbermann/Schaaf/Adam, 1980; Zum Instrumentarium der Filmanalyse s. Paech, 1975; Hickethier, 1981; Zu aktuellen Beispielen der Methodendiskussion s. Paech, 1984; Paech 1986).

Der vorliegende Band zum Thema >Literatur und Film< hat sich daher nicht auf den vordergründig dringendsten Bedarf der Schulund Hochschulpraxis nach Beispielanalysen und Methodendiskussionen zur Filmanalyse konzentriert, sondern versucht, einen Schritt weiterzugehen und >Film< im historisch-systematischen Kontext in seiner Beziehung zur Literatur vorzustellen. Daß Filmdidaktik mehr sein sollte als nur die ergänzende Diskussion einer Verfilmung zum literarischen Text, der primär behandelt wird, habe ich an anderer Stelle zu begründen versucht (Joachim Paech, Ist Film lehrbar? Plädoyer für Filmdidaktik in Schule und Universität, in: medien praktisch, Frankfurt 1987, H. 1 S. 4-8).

Gegenüber jeder Literaturgeschichte und auch einer $>$ Literaturgeschichte des Films wie der von Franz-Josef Albersmeier (1985) hat die Perspektive der Darstellung zugunsten des Films gewechselt: Ausgangspunkt sind die Anfänge des Films am Ende des vorigen Jahrhunderts. Damit die Bedingungen erkennbar werden, unter denen historisch und aus den jeweiligen Charakteristiken der beiden Medien Beziehungen zwischen ihnen möglich werden, war zunächst auf den (gegenüber der Literatur weitgehend) autonomen Ursprüngen des Films zu beharren, während andererseits von Anfang an die wesentliche Frage ist, wie Filme gelernt haben, zu erzählen. Die Literarisierung des Films seit etwa 1908/10 setzt voraus, daß Filme fähig sind, mit filmischen Mitteln literarisch Erzähl- 
tes wiederzugeben, so daß an diesem (historischen) Punkt auch (systematisch) die rudimentären Formen filmischer Artikulation erkennbar werden, die noch immer jedem Film zur Verfügung stehen.

Literarisierung bedeutet für den Film, den Zugang zur (bürgerlichen) Institution Kunst und Literatur zu suchen und zugleich ein unerschöpfliches Reservoir an erzählten Geschichten für das eigene, filmische Erzählen vorzufinden und in Anspruch zu nehmen, sowie die eigenen Fähigkeiten des Erzählens am Vorbild der Literatur ständig weiterzuentwickeln.

Aber die Beziehung zwischen Literatur und Film ist nie einseitig gewesen: Filmsehende Leser haben in den Romanen des 19. Jahrhunderts ,filmische Schreibweisen < bei Flaubert, Zola, Fontane u. a. entdeckt; Autoren, die auch ins Kino gegangen sind, haben angefangen, ihre filmischen Wahrnehmungen in ihre literarische Schreibweise einfließen zu lassen und schließlich auch für den Film geschrieben.

Ist diese Literatur, die unter dem Eindruck des Films und des Kinos entstanden ist, eine sneue Literatur<, die literarisch reflektiert, daß die audio-visuellen Medien dominanter Bestandteil der Lebensrealität und Kultur dieses Jahrhunderts geworden sind? Auch diese Frage ist in zwei Richtungen zu beantworten, da z. B. Drehbücher sowohl ganz traditionell wie Bühnendramen auch unabhängig von ihrer >Aufführung`gelesen und in der Tradition eines literarischen Genres verwendet wurden, als auch im avantgardistischen Sinne Beiträge zu einer >neuen< Literatur geliefert haben.

Film und Literatur haben unterschiedlich und widersprüchlich aufeinander reagiert: Zumal die Avantgarden beider Medien in ihrer Opposition gegenüber der etablierten (institutionalisierten) Literatur und Kunst versucht haben, sich im Falle des Films von literarischen Einflüssen freizuhalten oder sich im Falle der literarischen Avantgarde des Expressionismus, Surrealismus usw. ,filmisch $<$ zu geben, um gegen einen traditionellen Begriff von Literatur eine literarische Praxis zu setzen, die der Moderne mit ihren urbanen, dynamischen Lebensformen Ausdruck zu geben imstande ist. Der avantgardistische Stummfilm war hier der Malerei näher als der Literatur, während die Literatur in den ganz anderen Filmen der populären Genres von "Fantômas« bis "Dr. Mabuse« versuchte, den Massen und ihrer populären Kultur näher zu kommen.

Mit der Einführung des Tonfilms ist eine ganz neue Situation entstanden, da der Film von nun an tatsächlich >mitreden $<$ kann. Der (realistische) Tonfilm ist in einem Maße >literarisch $\varsigma$, daß er es schwer hat, sich von den Einflüssen der (realistisch erzählenden) 
Literatur freizuhalten und eigene, filmische Ausdrucksmöglichkeiten zu behaupten (Godard und die Nouvelle vague), während die Adaption literarischer Vorlagen zum Normalfall der Produktion für das Kino und das Fernsehen wird.

Diese diachrone Darstellung, die in Ausschnitten die Entwicklung der Beziehung zwischen Literatur und Film diskutiert, ist immer wieder durch synchrone Schnitte ergänzt worden, die versuchen, Stationen der Entwicklung von Formen filmischen Erzählens exemplarisch zu belegen. In der Literatur zur Filmtheorie und -geschichte gibt es noch kaum Werke, die sich mit der Entwicklung der Filmformen (etwa narrativer Stile) zufriedenstellend auseinandergesetzt haben - das bisher bedeutendste Werk stammt von Barry Salt (1983; außerdem: Peter and Sandra Klinge: Evolution of Film Styles. London 1983) -, da die Probleme durch die Vielzahl der Bedingungen in technischen, institutionellen, gesellschaftlichen, künstlerischen etc. Bereichen sehr groß sind. Das exemplarische Vorgehen und die Demonstration der Entwicklung von narrativen Strukturen an einer Reihe von Beispielen ermöglicht zumindest dort, wo die jeweiligen Filme erreichbar sind, den einen oder anderen Schritt in dieser formalen Entwicklung nachzuvollziehen.

Am Ende dieses Bandes steht ausdrücklich die Diskussion eines Beispiels für die Adaption einer Erzählung, die jeweils von einem Film und einem Roman realisiert wurde. Nicht ohne Hintergedanken und in der Perspektive des Films wurde der noch nicht allgemein übliche Fall gewählt, daß der Film die primäre, der Roman die sekundäre Realisierung ist, d. h. der Roman von Gerhard Zwerenz ist >nach dem Film »Die Ehe der Maria Braun« von R. W. Fassbinder entstanden. Die Analyse der literarischen >Lektüre dieses Films wurde begonnen in der Hoffnung, daß sie Interesse zur Fortsetzung weckt; dabei könnte deutlich werden, wie schwer es für die Adaption ist, dem Original gerecht zu werden - gleich, ob es sich um eine filmische oder wie hier um eine literarische Adaption handelt.

Überhaupt möchte dieses Buch Ansatzpunkte für eine viel weitergehende kompetente historische, theoretische und analytische Auseinandersetzung mit den audio-visuellen Medien geben, wie das literaturwissenschaftlich gegenüber der Literatur seit langem üblich ist und auch für den Film selbstverständlich werden sollte. 